\title{
TIME AND SPACE RESOLVED HEAT TRANSFER MEASUREMENTS UNDER NUCLEATE BUBBLES WITH CONSTANT HEAT FLUX BOUNDARY CONDITIONS
}

\author{
(1) National Aeronautics and Space Administration \\ Glenn Research Center \\ Microgravity Science Division \\ Brookpark, Ohio \\ (2) Department of Mechanical Engineering \\ University of Maryland \\ College Park, Maryland
}

Jerry G. Myers (1), Sam W. Hussey (1), Glenda F. Yee (1), and Jungho Kim (2)

\section{INTRODUCTION}

Investigations into single bubble pool boiling phenomena are often complicated by the difficulties in obtaining time and space resolved information in the bubble region. This usually occurs because the heaters and diagnostics used to measure heat transfer data are often on the order of, or larger than, the bubble characteristic length or region of influence. This has contributed to the development of many different and sometimes contradictory models of pool boiling phenomena and dominant heat transfer mechanisms.

Recent investigations by Yaddanapyddi and Kim [1] and Demiray and Kim [2] have obtained time and space resolved heat transfer information at the bubble/heater interface under constant temperature conditions using a novel micro-heater array ( $10 \times 10$ array, each heater 100 microns on a side) that is semi-transparent and doubles as a measurement sensor. By using active feedback to maintain a state of constant temperature at the heater surface, they showed that the area of influence of bubbles generated in FC-72 was much smaller than predicted by standard models and that microconduction/micro-convection due to re-wetting dominated heat transfer effects.

This study seeks to expand on the previous work by making time and space resolved measurements under bubbles nucleating on a micro-heater array operated under constant heat flux conditions. In the planned investigation, wall temperature measurements made under a single bubble nucleation site will be synchronized with high-speed video to allow analysis of the bubble energy removal from the wall.

\section{EXPERIMENTAL APPARATUS}

\section{Heater Array}

The heater array in this experiment is similar to those used by Demiray and Kim [2], with construction following the procedures outlined by Rule and Kim [3]. The array consists of 96 platinum resistance heaters deposited in a $10 \times 10$ configuration on a quartz wafer/substrate (Figure 1). Each array element is square with a nominal area of $0.01 \mathrm{~mm}^{2}$ and

This is a preprint or reprint of a paper intended for presentation at a conference. Because changes may be made before formal publication, this is made available with the understanding that it will not be cited or reproduced without the permission of the author. consists of $2 \mu \mathrm{m}$ wide Pt lines spaced $2 \mu \mathrm{m}$ apart. Each heater exhibits a nominal resistance of $6 \mathrm{k} \Omega$ and a temperature coefficient of resistance of $10 \Omega{ }^{\circ} \mathrm{C}^{-1}$. Gold lines that supply power to the heaters are routed between the heaters.

\section{Control Circuit}

Each heater is supplied with constant power by individual circuits. Each circuit consisted of a Wheatstone bridge and amplifier. During operation the analog output from each of the amplifiers is proportional to the voltage applied to the bridge and a simple resistance divider. This ensures that the amplifier output changes with respect to the heater resistance and that the heaters maintain a constant power input as their resistance varies with temperature.

Twenty-four circuits were constructed on a single card, thus requiring 4 cards to control the array. All 4 cards are connected to a custom designed multiplexer board that controls the voltage supply to the control cards and acts as router between each individual heater circuit and the computer/DAQ system.

\section{Heater Calibration}

Calibration of the heater array is performed in a two-step process. Initially the variation of heater resistance with temperature is determined by placing the heater array in an oven held to within $0.1{ }^{\circ} \mathrm{C}$ of the set temperature and measuring the resistance of each heater in the array over a range of temperatures from $40{ }^{\circ} \mathrm{C}$ to $100{ }^{\circ} \mathrm{C}$. A thermistor (Thermometrics Inc., MS type) attached to the underside of the substrate is used to insure stability of the temperature environment. Figure 2 illustrates the results of this calibration step for four selected heaters on the array.

The second step in the calibration involves calibrating measured output voltage from each heater circuit (relative to the applied power) with the resistance of the heater. In this calibration step, the control boards and multiplexer are connected to a banks of known resistors (nominally 6, 7 and 8 $\mathrm{k} \Omega$ ). The output voltages and data acquisition readings of each heater circuit are then measured at each resistance level. 
Since an amplifier's output voltage is directly correlated to the resistance of a heater, this two-step process provides measurements of heater temperature with an expected uncertainty less than $1^{\circ} \mathrm{C}$.

\section{Boiling Rig}

The boiling rig was custom designed and built at the NASA Glenn Research Center (illustrated in Figure 3) to handle FC-72, which is used as the working fluid in this experiment. The boiling chamber is a rectangular $9 \times 9 \times 14 \mathrm{~cm}$ aluminum chamber with a bellows pressure control system. The chamber incorporates three $5 \mathrm{~cm}$ diameter view ports to allow optical access to the heater array. External surface mounted heaters (Thermofoil, Minco Products, Inc.) are attached to the chamber and covered by foam insulation to control the bulk fluid temperature. Fluid access ports are placed on one side of the chamber to allow mixing with an external pump if stratification is detected. Bulk fluid temperature is measured by two T-type thermocouples placed at different heights in the chamber. Chamber pressure is measured by an absolute pressure transducer $(0-345 \mathrm{kPa},+/-$ $0.4 \mathrm{kPa}$ ) through an access port on one side of the chamber.

\section{Data Acquisition and High Speed Video}

A single data acquisition card (PCI-DIO96, Measurement Computing Corp.) capable of scanning 96 channels at $200 \mathrm{kHz}$ was installed in a Compaq Deskpro (Pentium 3-666MHz) computer and connected directly to the custom multiplex board. Custom software, written in $\mathrm{C}$, was created for experiment control. The combined system is capable of obtaining time resolved temperature data from each heater at a rate of $1890 \mathrm{~Hz}$ for a set period of time (usually less than 10 seconds).

Temperature acquisition is synchronized with high-speed video acquisition. Because of the semi-transparent nature of the heater and substrate, it is possible to acquire images of the bubble from below the boiling chamber using a high-speed camera (Vision Research Phantom V) set to acquire 512 5512 resolution images. Side view images of the bubble are obtained (256×256 resolution) using a second high-speed camera (Vision Research Phantom IV). Through the system multiplexer, the high-speed cameras are synchronized to acquire an image during the same rising edge TTL pulse from the computer. Two banks of high performance LED's provide the light for each camera view.

\section{Data Reduction and Numerical Simulation of Substrate Heat Conduction}

Although constant power is supplied to each heater, it is unclear what fraction is transferred to the fluid during the boiling process and what fraction is lost due to substrate conduction and eventually natural convection to the bulk fluid. In order to accurately estimate the heat lost due to substrate conduction, a numerical simulation is performed using
FEMLAB (Comsol Inc.), a multi-physics finite element software package. At the heater array location on the upper surface of the substrate, the experimentally measured heater array temperature field is used as a temporally varying Dirichlet boundary condition after being fit with a piecewise cubic spline to maintain temporal resolution. The remaining part of the substrate upper surface is assumed to exhibit a constant heat transfer coefficient, estimated from the average heater/bulk fluid temperatures. All other surfaces (bottom and sides) are assumed to have an insulated boundary condition. The simulation model geometry and associated boundary conditions are illustrated in Figure 4. Once completed, the simulation outputs the calculated substrate temperature field at each experimental acquisition time, from which the average conduction heat transfer of each heater into the substrate can be estimated. Once this is subtracted from the total heat flux of each heater, the heat flux into the bulk fluid can be obtained.

\section{PLANNED ACTIVITIES}

The boiling rig will utilize distilled/degassed FC-72 as the working fluid. Plans are to perform tests at several heat flux settings and several levels of bulk fluid subcooling. Initial tests will utilize bulk fluid temperatures of $\sim 52{ }^{\circ} \mathrm{C}$ and total heat flux levels supplied to the array ranging from 2 to 10 $\mathrm{W} / \mathrm{cm}^{2}$.

\section{REFERENCES}

1. Yaddanapudi, N., and Kim, J., "Single Bubble Heat Transfer in Saturated Pool Boiling of FC-72", Multiphase Science and Technology, Vol. 12, No. 3-4, pp. 47-63, 2001.

2. F. Demiray and J. Kim, Heat Transfer from a Single Bubble Nucleation Site During Saturated Pool Boiling of FC-72 Using an Array of 100 Micron heaters. 8th AIAA/ASME Joint Thermophysics and Heat Transfer Conference, June 2002.

3. T.D. Rule and J. Kim (1999,) Heat Transfer Behavior on Small Horizontal Heaters During Pool Boiling of FC-72, Journal of Heat Transfer, 121, No. 2, pp 386-393. 


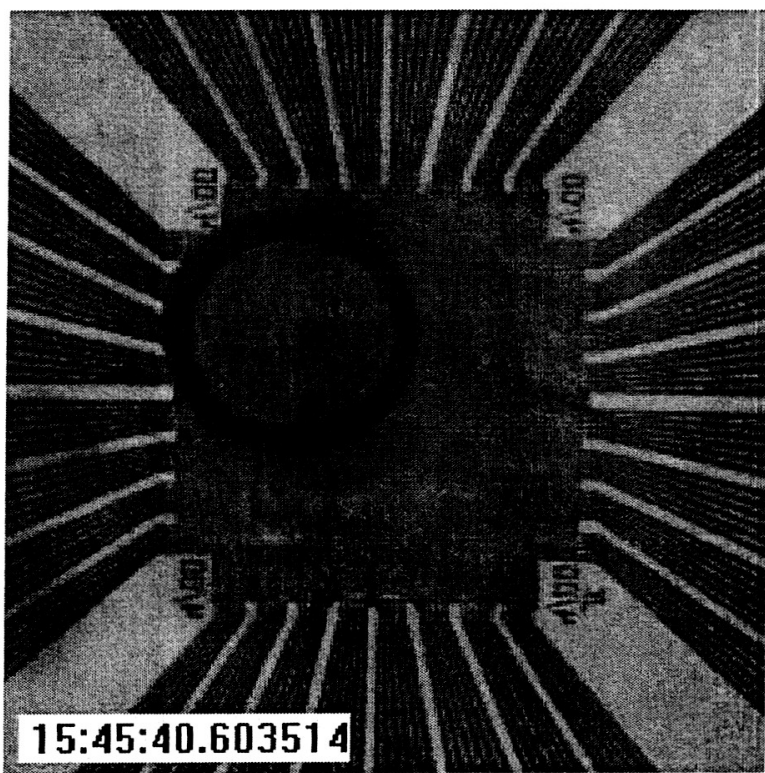

Figure 1: Photograph of heater array with single bubble nucleating on surface as seen from below the heater.

Constant power was supplied to the heater.

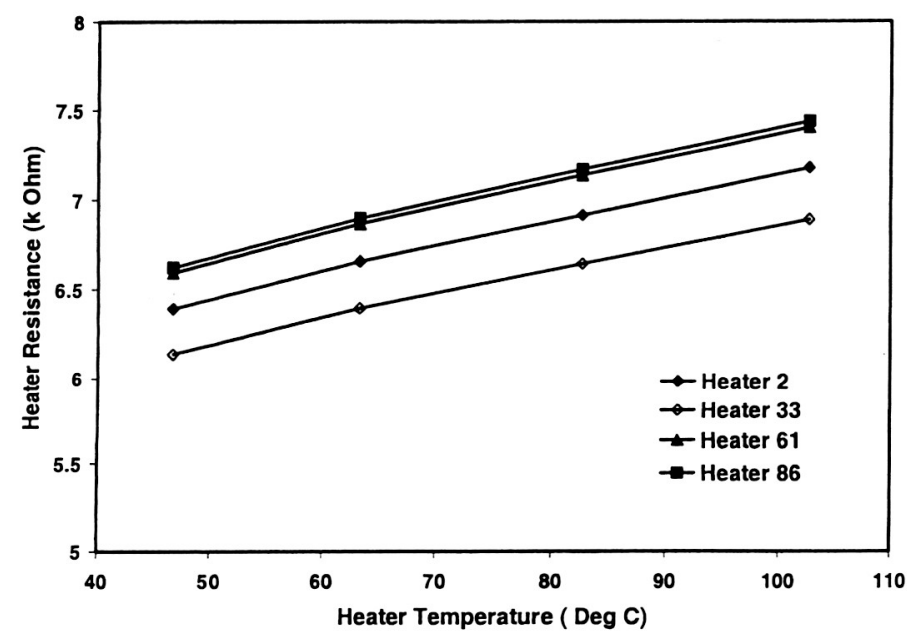

Figure 2: Plot of Heater resistance versus temperature calibration results for four selected heaters (one in each quadrant of the heater array).

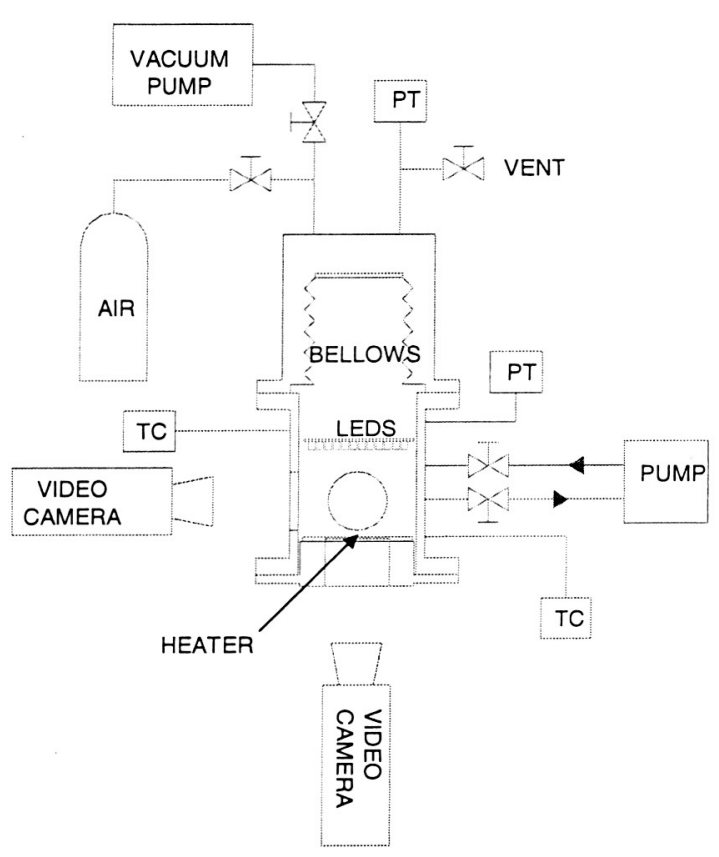

Figure 3: Illustration of custom designed boiling rig.

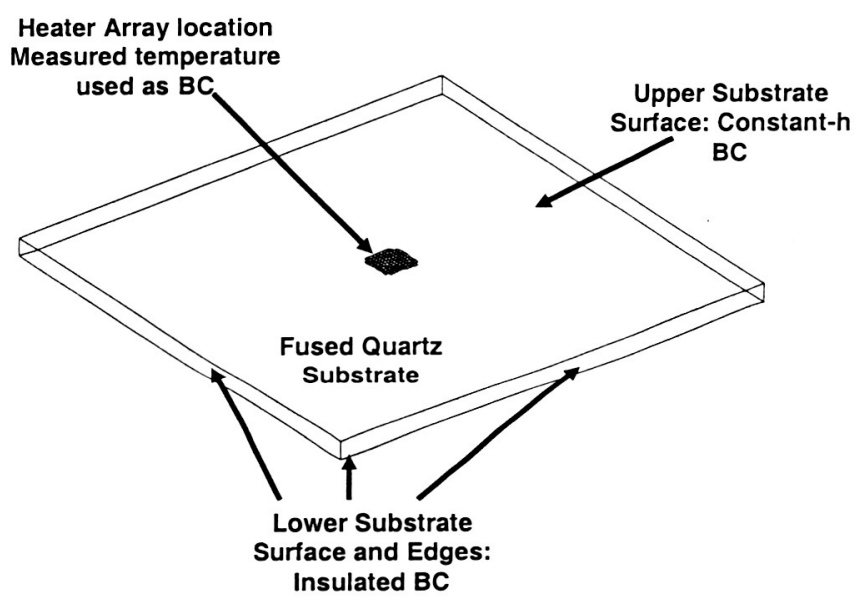

Figure 4: Illustration of substrate numerical model geometry and associated surface boundary conditions. The substrate is a fused quartz material approximately $0.5 \mathrm{~mm}$ thick and $6 \mathrm{~mm}$ on a side. 